\title{
Melanom: Je später die Operation, desto höher die Mortalität
}

\author{
Wird bei Patienten mit einem Melanom der Haut im Frühstadium nach der Biopsie die \\ Zeit bis zur Operation hinausgezögert, erhöht das möglicherweise die Sterberate. Eine \\ entsprechende Assoziation hat jetzt die Auswertung von US-Registerdaten ergeben.
}

Im Gegensatz zur Situation bei Patienten mit Brustkrebs oder mit einem Kopf-Hals-Tumor war bisher unklar, ob es Folgen hat, wenn bei Melanompatienten nach einer Biopsie nicht sofort chirurgisch behandelt wird. Melanomstudien, die es dazu bereits gibt, waren für aussagekräftige Ergebnisse zu klein. Deshalb haben US-amerikanische Dermatologen um Dr. Ruzica Z. Conic von der Cleveland Clinic Foundation in Cleveland Daten der National Cancer Database ausgewertet. Das Register enthält Befunde von fast jedem zweiten Melanompatienten in den USA. Die für die Studie ausgewerteten Daten stammten von 153.218 Patienten mit einem Melanom der Haut im TNM-Stadium I bis III. Das Follow-up lag zwischen 3 und 10 Jahren.

Wie Conic und ihre Kollegen berichten, wurden 71.950 Patienten frühestens 30 Tage nach der Biopsie operiert. Im Einzelnen lag der Anteil der Patienten im Stadium I, die erst nach 29 Tagen oder später so behandelt wurden, bei knapp $45 \%$. In den Stadien II und III waren das mit 50,3\% beziehungsweise 51,3\% deutlich mehr. Dagegen lag der Anteil der Patienten im Stadium I der Erkrankung, die frühestens nach 59 Tagen operiert wurden, nur bei $9 \%$.

\section{Später Eingriff vor allem bei Älteren und bei Männern} Wiederum deutlich mehr waren es in der Gruppe der Patienten im Stadium II (11,8\%) beziehungsweise III (11,7 \%). Patienten mit später chirurgischer Versorgung waren im Vergleich zu Patienten mit baldiger Operation nach der Biopsie eher älter und männlich und hatten außer dem Melanom noch andere Erkrankungen. Außerdem hatten sie Melanome mit höherer Breslow-Dicke und befanden sich bereits in einem höheren Erkrankungsstadium.

Schließlich geht aus der Multivariatanalyse der Daten hervor, dass Patienten, die erst zwischen 90 und 119 Tagen nach der Biopsie beziehungsweise noch später operiert wurden, ein erhöhtes Sterberisiko hatten (Hazard Ratio [HR]: 1,09 bzw. 1,12): Im Vergleich zu Patienten, die innerhalb von vier Wochen behandelt wurden, war das Risiko um $9 \%$ beziehungsweise $12 \%$ erhöht.

Die Folgen einer Verzögerung der chirurgischen Behandlung machen sich der Studie zufolge besonders bei Patienten im Stadium I bemerkbar. So ist das Sterberisiko um $16 \%$ erhöht (HR: 1,16; $95 \%$-Konfidenzintervall: 1,07-1,25), wenn erst 60 bis 89 statt spätestens 30 Tage nach der Biopsie operiert wird. Es ist sogar um $29 \%$ (HR: 1,29; $95 \%$-Konfidenzintervall: 1,12-1,48)

bzw. 41 \% (HR: 1,41; $95 \%$-Konfidenzintervall: 1,21-1,65) erhöht, wenn nach der Biopsie zwischen 90 und 119 Tage oder sogar noch länger mit dem Eingriff gewartet wird. Wenn die Patienten bereits im Stadium II oder III waren, hatte eine Verzögerung der Operation offenbar keinen signifikanten Einfluss auf das Überleben.

Nach Ansicht der Dermatologen sprechen auch diese Studienergebnisse dafür, Melanome möglichst schon im Frühstadium zu erkennen und zu behandeln, um so das Gesamtüberleben zu verbessern.

Peter Leiner

\section{Literatur}

Conic RZ et al. J Am Acad Dermatol 2017; http://dx.doi.org/10.1016/j. jaad.2017.08.039

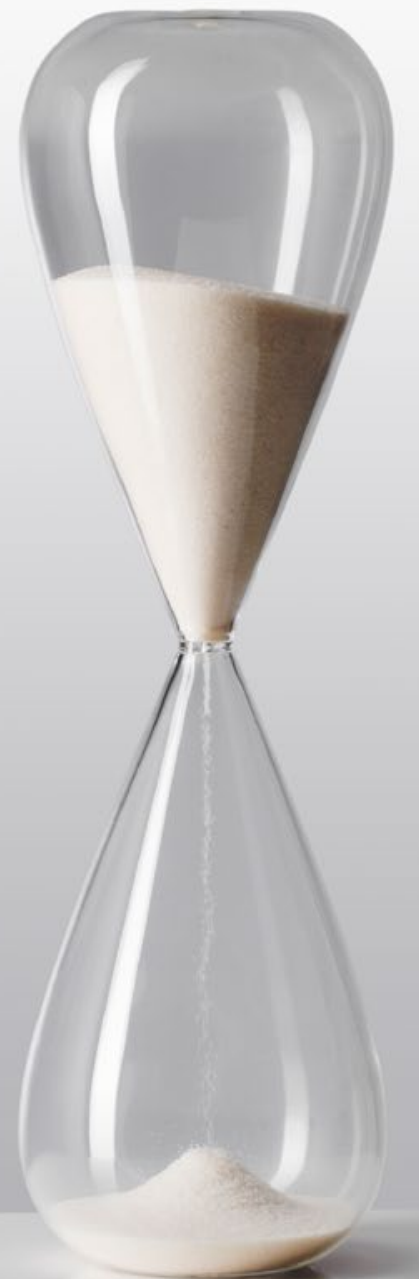

